\title{
A noninflammatory pathway for pregnancy loss: innate immune activation?
}

\begin{abstract}
Jane E. Salmon
Although the mechanisms of immune-mediated pregnancy loss are unknown, investigations are currently focused on mediators of immune activation and tissue injury at the maternal-fetal interface. A new study, however, demonstrates that systemic inflammatory mediators can induce pregnancy failure in a different way, by inhibiting ovarian hormone production, and identifies links between the immune and reproductive endocrine systems (see the related article beginning on page 39).
\end{abstract}

Department of Medicine, Hospital for Special Surgery, Weill Medical College of Cornell University, New York, New York, USA.
The journey from conception to birth is fraught with danger. It has been estimated that $50-70 \%$ of all conceptions fail and that recurrent pregnancy loss affects $1-3 \%$ of couples. The triggers and mediators of placental and fetal damage, however, are still poorly understood. When well-established genetic, anatomic, endocrine, and infectious causes of fetal damage are not demonstrable, as is the case in a majority of pregnancy complications, abnormal maternal immune responses are assumed to act as initiators of disease.

Evidence from murine and human pregnancy studies points to a strong association between maternal Th2-type immunity and successful pregnancy, whereas Th1type immune reactivity is associated with pregnancy loss (1). The proinflammatory Th1-dominant response that underlies clinical pregnancy failure is dependent on immunologic factors that may be amplified by environmental stimuli, such as lipopolysaccharides, autoantibodies, and stress. Murine studies have suggested that immune-mediated pregnancy failure is a consequence of immune activation at the maternal-fetal interface (Figure 1, left). Experimental models of miscarriage have focused on the placental milieu and show that pregnancy survival requires inhibition of local mediators of tissue damage. Complement-inhibitory proteins, maternal regulatory $\mathrm{T}$ cells, tryptophan-catabolizing enzymes, and immunoregulatory cytokines present at the maternal-fetal interface are

Nonstandard abbreviations used: suppressor of cytokine signaling (Socs).

Conflict of interest: The author has declared that no conflict of interest exists.

Citation for this article: J. Clin. Invest. 114:15-17 (2004). doi:10.1172/JCI200422258. thought to maintain maternal tolerance (2-4). In this issue of the JCI, Erlebacher et al. describe an alterative means by which immune activation can lead to pregnancy failure: inhibition of the reproductive endocrine system (5). In their model, systemic inflammatory mediators induce abortion by interfering with ovarian function, rather than by directly injuring the fetal-placental unit (Figure 1, right).

\section{Innate immune mediators at the maternal-fetal interface: local inflammation leads to pregnancy loss}

Pregnancy constitutes a major challenge to the maternal immune system, which must tolerate fetal alloantigens encoded by paternal genes. Local factors at the maternal-fetal interface are required to maintain such tolerance and to assure fetal survival. A growing body of evidence supports the concept that the adaptive immune response is regulated by the innate immune system; yet once engaged, adaptive immune responses can harness innate effectors to induce injury. Indeed, perturbations of innate immune responses or their regulators are associated with abortion, and complement activation, in particular, has emerged as a common causative element in recurrent fetal loss (6).

Activation of the complement cascade generates complement fragments that can bind to tissues, recruit potent effector cells, trigger inflammation, and cause injury (Figure 1). Trophoblasts express high levels of complement-inhibitory proteins to control complement-mediated damage (7). The importance of complement activation in pregnancy loss is underscored by murine studies showing that deficiency of complement-regulatory proteins produces extensive C3 deposition, neutrophil influx, and inflammation within the developing placenta that lead to embryonic death (2). Excessive complement activation and neutrophil infiltration are also the critical innate immune effectors engaged by antiphospholipid antibodies localized to decidual tissues in the antiphospholipid syndrome $(8,9)$. Inhibition of complement activation protects pregnancies from antiphospholipid antibody-induced fetal death or growth restriction. Even antibody-independent, T cell-dependent fetal allograft rejection is associated with complement activation and influx of neutrophils (3) (Figure 1). It is likely that uterine NK cells use similar mechanisms to directly trigger complement activation, perhaps through the alternative pathway (10).

While activation of complement and recruitment of neutrophils within decidual tissue are necessary intermediary steps in murine models of immune-mediated pregnancy loss, there are other downstream pathogenic mediators of placental and fetal damage. For example, studies of murine models of myocarditis, sepsis, and rheumatoid arthritis suggest that tissue damage induced as a result of complement activation is mediated by production of TNF- $\alpha$ (11-13), and increased placental levels of TNF- $\alpha$ have been associated with pregnancy failure in mice (refs. 14, 15; G. Girardi and J.E. Salmon, unpublished observations). Activation of immune cells within deciduae and the attendant release of cytokines have implications beyond local damage; they may also directly and indirectly influence the hormonal milieu (16-18).

\section{Systemic inflammation causes ovarian failure and pregnancy loss without local inflammatory changes}

The study by Erlebacher et al. identifies a new link between the immune system and the reproductive endocrine system (5). The authors show that systemic immune activation by CD40 ligation early in pregnancy inhibits the hypothalamic-pituitarygonadal axis and causes pregnancy failure without inflammatory injury in decidual 


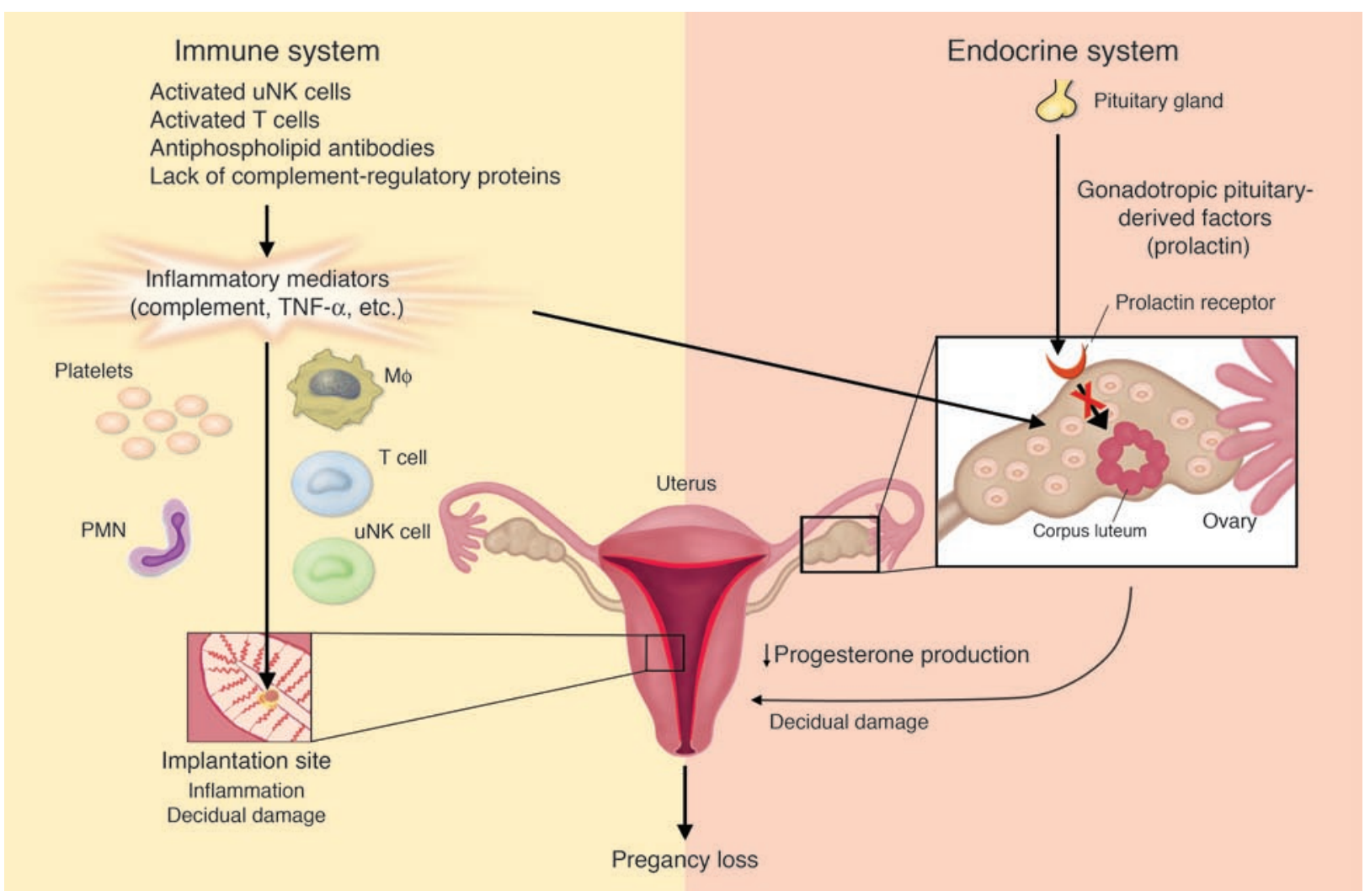

\section{Figure 1}

Inflammatory-mediated fetal damage (left): Activation of local inflammatory mediators by uterine NK (uNK) cells, T cells, antiphospholipid antibodies, or by uncontrolled amplification of the alternative pathway of complement leads to decidual damage and fetal injury or death, by initiating activation of the complement cascade and/or generating proinflammatory cytokines. These mediators directly or indirectly activate effector cells, including polymorphonuclear neutrophils (PMNs), macrophages $(\mathrm{M} \phi)$, uNK cells, and platelets, to release reactive oxidants, proteolytic enzymes, chemokines, cytokines, and thrombogenic factors. Depending on the extent of damage and stage of gestation, either death in utero or fetal growth restriction ensues. Inflammatory-mediated luteal insufficiency (right): These same initiators may induce fetal loss without decidual inflammation. Instead, systemic immune activation can trigger release of cytokines, such as TNF- $\alpha$, and other mediators, which cause ovarian resistance to gonadotropic pituitary-derived factors, such as prolactin in the mouse. Pregnancy loss results from inadequate progesterone synthesis.

tissues (5). In their model, embryos were resorbed as a result of cytokine-induced hormonal failure at the level of the ovary. NK cells and TNF- $\alpha$ were the critical effectors responsible for the induction of ovarian resistance to gonadotropic pituitaryderived factors that caused inadequate progesterone synthesis. Neither T cells nor B cells were required. Though the cellular and molecular mechanisms of luteal insufficiency in this model are likely multifactorial, there was clear evidence that systemic inflammation induced suppressor of cytokine signaling (Socs) proteins, which inhibited prolactin signaling and progesterone production by the ovary. That Socs induction and pregnancy resorption were partially prevented by TNF- $\alpha$ blockade implicates this sequence as a mechanism by which innate immune activation triggers a noninflammatory pathway for pregnancy loss.

\section{Implications for treatment}

What are the potential clinical implications of these studies? Although progesterone production is not regulated by prolactin in humans, the findings of Erlebacher and colleagues (5) emphasize that inflammatory mediators have important physiologic effects beyond influencing cells that mediate inflammation. They describe and define a novel interaction between the innate immune system and the reproductive endocrine system that, if operative in humans, would be a target for treatment in women with recurrent early miscarriage, independent of the triggers of immune activation (5) (Figure 1).

Blockade of systemic inflammatory mediators, such as TNF- $\alpha$, may prevent early pregnancy loss through two independent pathways: by reversing blockade of ovarian hormone production and by limiting inflammatory damage to the fetal- maternal unit. TNF- $\alpha$ has been implicated in pregnancy loss, since patients who have miscarried have elevated TNF- $\alpha$ levels (19) and are more likely to have TNF- $\alpha$ promoter alleles associated with increased cytokine levels (20). Blockade of TNF- $\alpha$ prevented abortion in murine models of stress-induced miscarriage early in pregnancy (15), but neutralization of TNF- $\alpha$ only partially reversed upregulation of ovarian Socs synthesis. Certainly, TNF- $\alpha$ is not the only cytokine known to inhibit luteal function (18). Furthermore, different initiators and mediators of pregnancy loss are likely to function at different stages of gestation. That the abortifacient effect of CD40 ligation was restricted to the immediate postimplantation period implies that targeted therapies may have narrow windows of efficacy.

Erlebacher et al. (5) have added to the conceptual framework within which ratio- 
nal therapeutic strategies can be developed for patients with recurrent early pregnancy loss. Therapies directed at innate inflammatory mediators, such as complement and TNF- $\alpha$, merit more intense scrutiny in pregnant women.

\section{Acknowledgments}

Work in the author's laboratory is supported by grants from the NIH (AR49772; AI55007), the Mary Kirkland Center for Lupus Research, and the Alliance for Lupus Research. Guillermina Girardi's critical review of the manuscript and invaluable help with the figures are gratefully acknowledged.

Address correspondence to: Jane E. Salmon, Hospital for Special Surgery, 535 East 70th Street, New York, New York 10021, USA. Phone: (212) 606-1422; Fax: (212) 717-1192; E-mail: salmonj@hss.edu.

1. Mellor, A.L., and Munn, D.H. 2000. Immunology at the maternal-fetal interface: lessons for $T$ cell tolerance and suppression. Annu. Rev. Immunol. 18:367-391.

2. Xu, C., et al. 2000. A critical role for murine complement regulator crry in fetomaternal tolerance.
Science. 287:498-501.

3. Mellor, A.L., et al. 2001. Prevention of T cell-driven complement activation and inflammation by tryptophan catabolism during pregnancy. Nat. Immunol. 2:64-68.

4. Aluvihare, V.R., Kallikourdis, M., and Betz, A.G. 2004. Regulatory $\mathrm{T}$ cells mediate maternal tolerance to the fetus. Nat. Immunol. 5:266-271.

5. Erlebacher, A., Zhang, D., Parlow, A.F., and Glimcher, L.H. 2004. Ovarian insufficiency and early pregnancy loss induced by activation of the innate immune system. J. Clin. Invest. 114:39-48. doi:10.1172/JCI200420645.

6. Caucheteux, S.M., Kanellopoulos-Langevin, C., and Ojcius, D.M. 2003. At the innate frontiers between mother and fetus: linking abortion with complement activation. Immunity. 18:169-172.

7. Tedesco, F., et al. 1993. Susceptibility of human trophoblast to killing by human complement and the role of the complement regulatory proteins. J. Immunol. 151:1562-1570.

8. Holers, V.M., et al. 2002. Complement C3 activation is required for antiphospholipid antibodyinduced fetal loss. J. Exp. Med. 195:211-220.

9. Girardi, G., et al. 2003. Complement C5a receptors and neutrophils mediate fetal injury in the antiphospholipid syndrome. J. Clin. Invest. 112:1644-1654. doi:10.1172/JCI200318817.

10. Schwaeble, W.J., and Reid, K.B. 1999. Does properdin crosslink the cellular and the humoral immune response? Immunol. Today. 20:17-21.

11. Wang, Y., et al. 2000. A role for complement in antibody-mediated inflammation: C5-deficient DBA/ 1 mice are resistant to collagen-induced arthritis. J. Immunol. 164:4340-4347.
12. Zwaka, T.P., et al. 2002. Complement and dilated cardiomyopathy: a role of sublytic terminal complement complex-induced tumor necrosis factoralpha synthesis in cardiac myocytes. Am. J. Pathol. 161:449-457.

13. Riedemann, N.C., et al. 2003. Regulation by C5a of neutrophil activation during sepsis. Immunity. 19:193-202.

14. Yui, J., Hemmings, D., Garcia-Lloret, M., and Guilbert, L.J. 1996. Expression of the human p55 and p75 tumor necrosis factor receptors in primary villous trophoblasts and their role in cytotoxic signal transduction. Biol. Reprod. 55:400-409.

15. Arck, P.C., Troutt, A.B., and Clark, D.A. 1997. Soluble receptors neutralizing TNF-alpha and IL-1 block stress-triggered murine abortion. Am. J. Reprod. Immunol. 37:262-266.

16. Mantzoros, C.S., et al. 1997. Leptin concentrations in relation to body mass index and the tumor necrosis factor-alpha system in humans. J. Clin. Endocrinol. Metab. 82:3408-3413.

17. Niswender, G.D., Juengel, J.L., Silva, P.J., Rollyson, M.K., and McIntush, E.W. 2000. Mechanisms controlling the function and life span of the corpus luteum. Physiol. Rev. 80:1-29.

18. Bornstein, S.R., Rutkowski, H., and Vrezas, I. 2004. Cytokines and steroidogenesis. Mol. Cell. Endocrinol. 215:135-141.

19. Raghupathy, R., et al. 2000. Cytokine production by maternal lymphocytes during normal human pregnancy and in unexplained recurrent spontaneous abortion. Hum. Reprod. 15:713-718.

20. Babbage, S.J., et al. 2001. Cytokine promoter gene polymorphisms and idiopathic recurrent pregnancy loss. J. Reprod. Immunol. 51:21-27.

\title{
Lonely in Paris: when one gene copy isn't enough
}

\section{Ramesh A. Shivdasani}

\begin{abstract}
Departments of Medical Oncology and Cancer Biology, Dana-Farber Cancer Institute; and Department of Medicine, Harvard Medical School,
\end{abstract} Boston, Massachusetts, USA.

\begin{abstract}
Circulating platelets are continually replenished by fragmentation of terminally differentiated megakaryocytes. Processes disrupted in inherited thrombocytopenias frequently shed light on normal thrombopoietic mechanisms. An especially rare condition called Paris-Trousseau syndrome (PTS) seems to occur by virtue of hemizygous loss of the FLI1 transcription factor gene. Provocative new data suggest that FLI1 shows monoallelic expression during a brief window in megakaryocyte differentiation, which thus explains the dominant inheritance pattern of PTS despite the presence of one normal FLI1 allele (see the related article beginning on page 77).
\end{abstract}

The rare familial thrombocytopenic disorder Paris-Trousseau syndrome (PTS), reported in fewer than a dozen patients, is associated with inherited deletion of a portion of the long arm of chromosome 11 (1). Blood platelets in affected individ-

Nonstandard abbreviations used: GATA binding protein 1 (GATA-1); Paris-Trousseau syndrome (PTS).

Conflict of interest: The author has declared that no conflict of interest exists.

Citation for this article: J. Clin. Invest. 114:17-19 (2004) doi:10.1172/JCI200422292. uals have a normal lifespan in the circulation; however, the number of bone marrow megakaryocytes is greatly increased, whereas megakaryocyte maturation is arrested, and many cells apparently fail to survive into terminal stages of differentiation $(1,2)$. Together these findings suggest that platelet release is substantially impaired. Megakaryocyte release of newly synthesized platelets, a process unique to mammals, occurs through a dramatic morphogenetic transition $(3,4)$. Nearly the entire cytoplasm converts into lengthy beaded projections (proplatelets), within which organelles are transported along unique microtubule tracks and nascent blood platelets assemble de novo (5) (Figure 1). The use of thrombopoietin, a megakaryocyte growth factor, to expand cells ex vivo and incisive mouse models of thrombocytopenia has led to rapid progress in understanding genetic and cellular mechanisms of platelet biogenesis (6); the emerging appreciation has important implications for diseases in which hemorrhage or thrombosis is a significant feature. In this issue of the JCI, Raslova and colleagues highlight transient monoallelic expression of a transcription factor gene en route to platelet release and explain why patients with PTS, who retain one normal copy of chromosome 11, produce few and defective platelets (7). Related gene regulatory mechanisms might well account for other dominantly inherited disorders in which haploinsufficiency or 